\title{
Effect of Pretreatments on Seed Viability During Fruit Development of Two Varieties of Irvingia gabonensis
}

\author{
P. J. Nya*, D. N. Omokaro and A. E. Nkang \\ Department of Botany, University of Calabar, P.M.B. 1115, Calabar, Nigeria \\ *Corresponding author; E-mail: Akpauson@yahoo.com
}

\begin{abstract}
Studies to identify the stage at which developing fruits of Irvingia gabonensis (var. excelsa and var. gabonensis), picked from standing trees and/or forest floors, attain maximum viability and germinability were conducted in two harvesting seasons in 2000 and 2001. Some pretreatment methods were used as a means of stimulating expression of maximum germinability as source of seeds for seedling production. The moisture contents of whole seeds decreased steadily and significantly $(P=0.05)$ throughout the developmental stages of both varieties and in both harvests. Seeds harvested from standing trees and forest floors, pretreated by steeping in water for $24 \mathrm{~h}$ at $26^{\circ} \mathrm{C}$ and sown in sawdust and petri dishes on moist filter paper, gave $80 \%$ germination in variety gabonensis, 20 weeks after fruiting. Germination potentials of seeds in both harvests were enhanced significantly $(P=0.05)$ with seeds pretreated by steeping in water for $24 \mathrm{~h}$ at $26{ }^{\circ} \mathrm{C}$, and sown in sawdust and petri dishes on moist filter paper by as much as $60 \%$ and $80 \%$ for var. excelsa and var. gabonensis, respectively, in the developmental stages, 14-20 weeks after fruiting with significant $(P=0.05)$ decrease in germination time to 21 and 15 days, respectively. There was also significant $(P=$ 0.05) relationship between viability and germination in both harvests and in the two varieties throughout the developmental stages. Potassium nitrate $\left(\mathrm{KNO}_{3}\right)$ significantly $(P=0.05)$ enhanced germination potentials of the seeds over the control when soaked in $1.5 \mathrm{~g} / \mathrm{l}$ concentration in both varieties and in the two harvests (18-20 WAF) by $80 \%$. Mannitol significantly $(P=0.05)$ reduced germination potential $(40 \%)$ of the seeds with increased germination time in both harvests and in the two varieties throughout the developmental stages. There was a high significant $(P=0.05)$ increase in germination potential $(60 \%)$ of seeds treated with polyethylene glycol (PEG) when soaked in concentration of $10 \mathrm{~g} / 1$ for 48 and $72 \mathrm{~h}$ in both varieties and in the two harvests (18-20 WAF). The study shows that the fruits of $I$. gabonensis assume ripening characteristics (yellowing) and that the viable seeds can be obtained from standing trees and/or picked from forest floors from the 12th to 20th week after fruiting.
\end{abstract}

\section{Introduction}

Irvingia gabonensis (bush mango) is one of the most economically viable forest tree species in the rain forest zone of West Africa. It is a source of food, income, an essential raw material for the pharmaceutical industries and, above all, a potential foreign exchange earner (Okafor, 1989; Okafor et al., 1994; Omokaro et al., 1999; Omoluabi, 1994). In recognition of its importance, the Federal Government of Nigeria, through her Agency, Directorate for Food, Road and Rural Infrastructure, has shown great interest in the development of I. gabonensis plantation (Oni, 1984; Adeyoju \& Enabor, 1985). Efforts so far made have met with little success because the only successful propagation method (budding) presently used in seedling production is not adequate for rapid multiplication of seedlings. Besides, I. gabonensis seeds exhibit poor germination potential with less than $50 \%$ germination capacity when freshly collected (Nya et al., 2000).

In the local communities of Cross River State, Nigeria, where I. gabonensis trees are found growing in the wild, fruits from standing trees and forest floor can be harvested if permitted by the community (Nya, 1997). In order to help establish this viable tree species as a plantation crop, the study was undertaken to investigate the stage of development when mature fruits on standing trees, or falling fruits from forest floors, attain maximum germinability and also to determine pretreatment methods that may stimulate expression of maximum germinability.

Seed collection and treatment

\section{Materials and methods}

Five hundred fruits of each variety of Irvingia gabonensis (var. excelsa and var. gabonensis) were harvested between December 2000 and June 2001 from standing trees of age range between 
20 and 30 years or forest floor at Abredang Abeyong rain forest zone located south-west of Ugep, in Cross River State of Nigeria (latitude 05 44' $14^{\prime \prime} \mathrm{N}$, longitude $08^{\circ} 02^{\prime} 25^{\prime \prime} \mathrm{E}$ ). The harvesting was started 10 weeks from commencement of fruiting and continued at forthnightly intervals until the 20th week. At each harvest, 50 freshly plucked or picked fruits of each variety (standing tree and forest floor, respectively) of approximately the same size were immediately depulped to extract the seeds. Seeds extracted from both harvests were used for moisture content (MC) determination. Viability tests using tetrazolium and germination studies were carried out.

\section{Determination of moisture content}

Moisture content (MC) of whole seeds was determined on five randomly picked seeds from both harvests after oven-dried at $80{ }^{\circ} \mathrm{C}$ to constant weight. Moisture content was calculated on a fresh weight basis according to ISTA (1985 a b) as follows:

RMC (Relative moisture content $)=$

Fresh weight of seed - Dry weight of seed $\times 100$

Fresh weight of seed 1

\section{Triphenyl tetrazolium chloride (TZ) test for viability}

Ten seeds of each variety from both harvests were imbibed in distilled water for $12 \mathrm{~h}$ followed by dissection of the embryos into equal halves. Half of the dissected embryos were then placed in petri dishes containing $0.5 \%$ solution of TZ. The petri dishes were then wrapped in aluminium foil and incubated for $4 \mathrm{~h}$ after which the embryos were then washed and observed for red colouration. The coloured embryos were counted as viable. Percentage viability from five replicates were recorded (Demir \& Ellis, 1992).

\section{Seed pretreatments and germination tests}

Five seeds from each seed lots of the two harvests from freshly depulped fruits of each variety (standing tree and forest floor) were surface-sterilized with $0.1 \%$ mercuric chloride and then washed several times in sterile distilled water. The seeds were either sown in petri dishes on Whatman's filter paper (No. 1) or in polyethylene bags filled with moist decomposed sawdust. Similarly, batches of five seeds were pretreated by steeping in water for $24 \mathrm{~h}$ at ambient temperature $\left(26-28^{\circ} \mathrm{C}\right)$ before sowing as above.

Sixty seeds from both harvests of the two varieties were pretreated with different concentrations of $\mathrm{KNO}_{3}(1.5 \mathrm{~g} / \mathrm{l}$ and $3.0 \mathrm{~g} / \mathrm{l})$. Five seeds from each concentration were removed, after 24, 48 and $72 \mathrm{~h}$, washed with distilled water and immediately sown in moist decomposed sawdust. Another batch of seeds were also soaked in manitol with the same concentrations and sown as above.

Sixty seeds of both harvests were also pretreated by soaking in two concentrations of polyethylene glycol (PEG) $5 \mathrm{~g} / 1$ and $10 \mathrm{~g} / 1$ for 24,48 and $72 \mathrm{~h}$. The seeds were removed after these periods, washed and sown in moist sawdust. The petri dishes and polyethylene bags were arranged in a randomized complete block design with five replications on the laboratory bench and in the botanical garden of the University of Calabar, respectively.

Germination counts were made daily and recorded. The emergence of plumule and radicle protrusion $(2 \mathrm{~mm})$ were used as indicators of germination in moist decomposed sawdust and petri dishes, respectively. All data were subjected to an analysis of variance and the standard error (SE) of means were compared. Linear correlation and regression analysis were carried out in order to ascertain the level of relationship between viability and germinability during development.

\section{Results}


The results showed that any single parent tree of Irvingia gabonensis bears fruits of varying physiological maturity and/or viability in a fruiting season. All seeds of both varieties (var. gabonensis and var. excelsa) harvested from standing trees and forest floor in the first 10 weeks are physiologically immature and not viable (Tables 1 and 2).

TABLE 1

Effect of pretreatments on germination of seeds of fruits of Irvingia gabonensis plucked from standing trees (Mean + $S E)$

h) $\quad$ Imbibed seeds $(24 \mathrm{~h})$
$\left.{ }^{\circ} \mathrm{C}\right)$

Unimbibed seeds

sown in sawdust

$\left(26^{\circ} \mathrm{C}\right)$

\begin{tabular}{|c|c|c|c|c|c|c|c|c|c|}
\hline Germ. & $\begin{array}{l}\text { Harvest } \\
\text { Germ.\% }\end{array}$ & $\begin{array}{c}M C(\%) \\
\text { Germ. }\end{array}$ & Viability & Germ.\% & Germ. & Germ.\% & Germ. & Germ.\% & \\
\hline Variety & time & time & $T Z(\%)$ & & time & & time & & time \\
\hline (days) & (week) & (days) & & & (days) & & (days) & & \\
\hline \multirow[t]{2}{*}{0.0} & $\begin{array}{l}10 \\
0 \pm 0.0\end{array}$ & $\begin{array}{l}50.6 \pm 2.0 \\
0 \pm 0.0\end{array}$ & $0 \pm 0.0$ & $0 \pm 0.0$ & $60 *$ & $0 \pm 0.0$ & $0 \pm 0.0$ & $0 \pm 0.0$ & $0 \pm$ \\
\hline & 12 & $49.2 \pm 2.9$ & $40 \pm 1.2$ & $00 \pm 0.0$ & $0 \pm 0.0$ & $0 \pm 0.0$ & $0 \pm 0.0$ & $0 \pm 0.0$ & $0 \pm$ \\
\hline 0.0 & $0 \pm 0.0$ & $0 \pm 0.0$ & & & & & & & \\
\hline Var. & 14 & $48.5 \pm 3.0$ & $60 \pm 2.2$ & $00 \pm 0.0$ & $0 \pm 0.0$ & $0 \pm 0.0$ & $0 \pm 0.0$ & $20 \pm 2.6$ & $22 \pm$ \\
\hline 1.3 & $20 \pm 1.2$ & $23 \pm 1.3$ & & & & & & & \\
\hline gabonensis & 16 & $44.8 \pm 2.8$ & $80 \pm 0.2$ & $20 \pm 2.1$ & $25 \pm 1.2$ & $40 \pm 1.8$ & $26 \pm 0.9$ & $60 \pm 3.0$ & $18 \pm$ \\
\hline \multirow[t]{2}{*}{1.0} & $60 \pm 1.0$ & $18 \pm 1.0$ & & & & & & & \\
\hline & 18 & $36.6 \pm 2.4$ & $100 \pm 3.2$ & $40 \pm 3.1$ & $20 \pm 1.3$ & $40 \pm 4.0$ & $24 \pm 0.0$ & $60 \pm 1.7$ & $18 \pm$ \\
\hline \multirow[t]{2}{*}{1.7} & $80 \pm 4.0$ & $18 \pm 1.0$ & & & & & & & \\
\hline & 20 & $30.4 \pm 2.6$ & $100 \pm 3.1$ & $60 \pm 4.0$ & $20 \pm 1.0$ & $40 \pm 3.7$ & $24 \pm 0.7$ & $80 \pm 3.0$ & $17 \pm$ \\
\hline \multirow[t]{2}{*}{0.0} & $80 \pm 2.0$ & $15 \pm 0.0$ & & & & & & & \\
\hline & 10 & $45.1 \pm 2.0$ & $0 \pm 0.0$ & $0 \pm 0.0$ & $0 \pm 0.0$ & $0 \pm 0.0$ & $0 \pm 0.0$ & $0 \pm 0.0$ & $0 \pm$ \\
\hline \multirow[t]{2}{*}{0.0} & $0 \pm 0.0$ & $0 \pm 0.0$ & & & & & & & \\
\hline & 12 & $44.9 \pm 1.2$ & $40 \pm 1.2$ & $\begin{array}{ll}0 & 0.0\end{array}$ & $0 \pm 0.0$ & $0 \pm 0.0$ & $0 \pm 0.0$ & $0 \pm 0.0$ & $0 \pm$ \\
\hline 0.0 & $0 \pm 0.0$ & $0 \pm 0.0$ & & & & & & & \\
\hline Var. & 14 & $43.8 \pm 2.6$ & $80 \pm 2.3$ & $\begin{array}{ll}0 & 0.0\end{array}$ & $0 \pm 0.0$ & $0 \pm 0.0$ & $0 \pm 0.0$ & $40 \pm 2.0$ & $30 \pm$ \\
\hline 1.3 & $20 \pm 0.7$ & $33 \pm 2.0$ & & & & & & & \\
\hline excelsa & 16 & $41.7 \pm 1.2$ & $80 \pm 3.1$ & $20 \pm 3.0$ & $30 \pm 2.0$ & $40 \pm 1.2$ & $28 \pm 1.7$ & $60 \pm 3.1$ & $26 \pm$ \\
\hline \multirow[t]{2}{*}{1.4} & $40 \pm 1.2$ & $31 \pm 0.0$ & & & & & & & \\
\hline & 18 & $34.1 \pm 1.6$ & $80 \pm 3.3$ & $20 \pm 1.6$ & $26 \pm 1.3$ & $40 \pm 1.0$ & $28 \pm 1.2$ & $60 \pm 1.1$ & $25 \pm$ \\
\hline \multirow[t]{2}{*}{1.3} & $60 \pm 2.0$ & $29 \pm 1.3$ & & & & & & & \\
\hline & 20 & $29.6 \pm 2.0$ & $80 \pm 2.5$ & $40 \pm 2.3$ & $24 \pm 1.2$ & $40 \pm 1.2$ & $25 \pm 1.3$ & $60 \pm 2.0$ & $60 \pm$ \\
\hline 2.0 & $60 \pm 2.0$ & $24 \pm 0.3$ & & & & & & & \\
\hline
\end{tabular}

Unimbibed seeds sown Imbibed seeds (24 in moist filter paper sown in sawdust $(26$

e 
Key: $60^{*}=$ There was no germination 60 days after sowing; $\mathrm{MC}=$ Moisture content; Germ. $\%=$ Germination percentage; Germ. time $=$ Germination time.

Regression relationship: Viability on maximum germination, with steeping; $\mathrm{y}=139.8-2.55 \mathrm{x}$ with $r=0.71 \quad(P<$ $0.05)$.

TABLE 2

Effect of pretreatments on seed germination of fruits of Irvingia gabonensis plucked from forest floor (Mean + S.E)

${ }^{\circ}$ C)

sown in moist filter

Unimbibed seeds

sown in sawdust

$\left(26^{\circ} \mathrm{C}\right)$

\begin{tabular}{|c|c|c|c|c|c|c|c|c|c|}
\hline Germ. & $\begin{array}{l}\text { Harvest } \\
\text { Germ.\% }\end{array}$ & $\begin{array}{l}\text { MC (\%) } \\
\text { Germ. }\end{array}$ & Viability & Germ.\% & Germ. & Germ.\% & Germ. & Germ.\% & \\
\hline $\begin{array}{l}\text { Variety } \\
\text { time } \\
\text { (days) }\end{array}$ & $\begin{array}{l}\text { time } \\
\text { (week) }\end{array}$ & $\begin{array}{l}\text { time } \\
\text { (days) }\end{array}$ & $T Z(\%)$ & & $\begin{array}{l}\text { time } \\
\text { (days) }\end{array}$ & & $\begin{array}{l}\text { time } \\
\text { (days) }\end{array}$ & & \\
\hline .0 & $\begin{array}{l}10 \\
0 \pm 0.0 \\
12 \\
0 \pm 0.0\end{array}$ & $\begin{array}{r}52.4 \\
0 \pm 0.0 \\
48.4 \\
0 \pm 0.0\end{array}$ & $\begin{array}{l} \pm 1.140 \pm 2.1 \\
\pm 2.060 \pm 0.0\end{array}$ & $\begin{array}{l}0 \pm 0.0 \\
0 \pm 0.0\end{array}$ & $\begin{array}{r}0 \pm 0.0 \\
00 \pm 0.0\end{array}$ & $\begin{array}{l}0 \pm 0.0 \\
0 \pm 0.0\end{array}$ & $\begin{array}{l}0 \pm 0.0 \\
0 \pm 0.0\end{array}$ & $\begin{array}{l}0 \pm 0.0 \\
0 \pm 0.0\end{array}$ & $\begin{array}{l}00 \pm 0 \\
00 \pm 0\end{array}$ \\
\hline $\begin{array}{l}\text { Var. } \\
.2\end{array}$ & $\begin{array}{l}14 \\
40 \pm 2.1\end{array}$ & $\begin{array}{r}44.3 \\
21 \pm 1.6\end{array}$ & $\pm 2.280 \pm 0.2$ & $0 \pm 0.0$ & $00 \pm 0.0$ & $0 \pm 0.0$ & $0 \pm 0.0$ & $20 \pm 0.0$ & $17 \pm 0$ \\
\hline $\begin{array}{l}\text { gabonensis } \\
.2\end{array}$ & $\begin{array}{l}16 \\
60 \pm 2.1\end{array}$ & $\begin{array}{r}36.6 \\
19 \pm 0.8\end{array}$ & $\pm 1.280 \pm 2.32$ & $20 \pm 1.0$ & $23 \pm 1.2$ & $40 \pm 1.0$ & $34 \pm 1.0$ & $60 \pm 2.1$ & $18 \pm 0$ \\
\hline .1 & $\begin{array}{l}18 \\
60 \pm 2.1\end{array}$ & $\begin{array}{r}31.3 \\
18 \pm 0.5\end{array}$ & $\pm 2.0100 \pm 3.4$ & $440 \pm 2.0$ & $20 \pm 0.6$ & $40 \pm 3.1$ & $26 \pm 3.1$ & $80 \pm 3.1$ & $15 \pm 0$ \\
\hline .1 & $\begin{array}{l}20 \\
80 \pm 3.2\end{array}$ & $\begin{array}{r}29.2 \\
18 \pm 0.6\end{array}$ & $\pm 2.0100 \pm 4.1$ & $140 \pm 3.6$ & $16 \pm 0.4$ & $40 \pm 3.1$ & $26 \pm 3.1$ & $80 \pm 3.0$ & $15 \pm 0$ \\
\hline .0 & $\begin{array}{l}10 \\
0 \pm 0.0\end{array}$ & $\begin{array}{r}46.8 \\
0 \pm 0.0\end{array}$ & $\pm 1.440 \pm 0.0$ & $0 \pm 0.0$ & $0 \pm 0.0$ & $0 \pm 0.0$ & $0 \pm 0.0$ & $0 \pm 0.0$ & $0 \pm 0$ \\
\hline .8 & $\begin{array}{l}12 \\
20 \pm 0.5\end{array}$ & $\begin{array}{r}44.1 \\
36 \pm 1.0\end{array}$ & $\pm 2.160 \pm 2.1$ & $0 \pm 0.0$ & $0 \pm 0.0$ & $0 \pm 0.6$ & $0 \pm 0.0$ & $20 \pm 0.6$ & $37 \pm 0$ \\
\hline $\begin{array}{l}\text { Var. } \\
.6\end{array}$ & $\begin{array}{l}14 \\
40 \pm 0.1\end{array}$ & $\begin{array}{r}40.6 \\
35 \pm 1.0\end{array}$ & $\pm 1.480 \pm 3.3$ & $0 \pm 0.0$ & $0 \pm 0.0$ & $0 \pm 0.7$ & $0 \pm 0.0$ & $40 \pm 0.7$ & $31 \pm 0$ \\
\hline $\begin{array}{l}\text { excelsa } \\
.9\end{array}$ & $\begin{array}{l}16 \\
40 \pm 0.6\end{array}$ & $\begin{array}{r}35.7 \\
34 \pm 1.2\end{array}$ & $\pm 1.680 \pm 3.6$ & $0 \pm 0.0$ & $0 \pm 0.0$ & $0 \pm 0.6$ & $0 \pm 0.0$ & $40 \pm 0.8$ & $29 \pm 0$ \\
\hline .8 & $\begin{array}{l}18 \\
60 \pm 1.3\end{array}$ & $\begin{array}{r}30.6 \\
30 \pm 0.9\end{array}$ & $\pm 1.6100 \pm 2.5$ & $520 \pm 1.0$ & $29 \pm 1.4$ & $40 \pm 0.9$ & $27 \pm 1.0$ & $40 \pm 0.7$ & $27 \pm 0$ \\
\hline .7 & $\begin{array}{l}20 \\
60 \pm 2.1\end{array}$ & $\begin{array}{r}28.1 \\
26 \pm 0.5\end{array}$ & $\pm 1.6100 \pm 3.1$ & $140 \pm 1.0$ & $27 \pm 1.0$ & $40 \pm 1.0$ & $24 \pm 1.1$ & $60 \pm 1.3$ & $26 \pm 0$ \\
\hline
\end{tabular}

Unimbibed seeds sown $\quad$ Imbibed seeds (24 h) Imbibed seeds $(24 \mathrm{~h})$

in moist filter paper sown in sawdust

(26

Key: $60 *=$ There was no germination 60 days after sowing; $\mathrm{MC}=$ Moisture content; Germ. $\%=$ Germination percentage; Germ. time $=$ Germination time.

Regression relationship: Viability on maximum germination, with steeping; $\mathrm{y}=36.76-0.78 \mathrm{x}$ with $r=0.77(P<$ 0.05). 
The moisture contents of whole seeds were high in the immature seeds and this decreased significantly $(P<0.05)$ during development (Tables 1 and 2$)$. Results showed that un-imbibed seeds sown in sawdust, moist filter paper in Petri dishes recorded $40 \%$ germination 20 weeks after fruiting in both varieties at both harvests (Tables 1 and 2).

Pretreating whole seeds harvested from standing trees and forest floor by steeping in water for $24 \mathrm{~h}$ at $26{ }^{\circ} \mathrm{C}$ recorded $80 \%$ germination in both varieties 20 weeks after fruiting. Germination potentials of seeds in both harvests were enhanced significantly $(P<0.05)$ by treatment as much as $80 \%$ and $60 \%$ in varieties gabonensis and excelsa, respectively, in the develop-mental stages (16-20 weeks after fruiting). Pretreatment of seeds from both harvests by steeping in water for $24 \mathrm{~h}$ at $26^{\circ} \mathrm{C}$ resulted in a significant $(P<0.05)$ decrease in germination time to 15 and 21 days in varieties gabonensis and excelsa, respectively (Tables 1 and 2).

The results showed that there was a positive correlation and significant $(P<0.05)$ relationship between viability and germinability in both harvests throughout the developmental stages in the two varieties of Irvingia (Tables 1 and 2).

Pretreatment with $\mathrm{KNO}_{3}$ resulted in $80 \%$ germination in both varieties from seeds harvested from standing trees and forest floors between 18 and 20 WAF (Tables 3 and 4). The results also showed that the longer the soaking period, the higher the germination percentage with significantly $(P<0.05)$ reduced germination time (Tables 3 and 4$)$.

TABLE 3

Effect of potassium nitrate $\left(\mathrm{KNO}_{3}\right)$ on percentage germination of seeds of fruits of Irvingia varieties plucked from standing trees (Mean + S.E)

\begin{tabular}{|c|c|c|c|c|c|c|c|c|c|c|}
\hline Harvest & 3.0 & & $\begin{array}{r}\text { Var. gab } \\
\text { Concen }\end{array}$ & $\begin{array}{l}\text { nensis } \\
\text { ation }(g / l)\end{array}$ & 1.5 & Hours of & oaking & & & \\
\hline period & $\begin{array}{l}24 \\
72\end{array}$ & & 48 & & 72 & & 24 & & 48 & \\
\hline (week) & $\begin{array}{l}G \% \\
G \%\end{array}$ & $\begin{array}{l}G T \\
G T\end{array}$ & $G \%$ & $G T$ & $G \%$ & $G T$ & $G \%$ & $G T$ & $G \%$ & $G T$ \\
\hline 10 & $\begin{array}{l}0 \pm 0.0 \\
0 \pm 0.0\end{array}$ & $\begin{array}{l}0 \\
-\end{array}$ & $0 \pm 0.0$ & - & $0 \pm 0.0$ & - & $0 \pm 0.0$ & - & $0 \pm 0.0$ & - \\
\hline 12 & $\begin{array}{l}0 \pm 0.0 \\
0 \pm 0.0\end{array}$ & $\begin{array}{l}- \\
-\end{array}$ & $0 \pm 0.0$ & - & $0 \pm 0.0$ & - & $0 \pm 0.0$ & - & $0 \pm 0.0$ & - \\
\hline $\begin{array}{l}14 \\
0.5\end{array}$ & $\begin{array}{l}20 \pm 2.0 \\
20 \pm 1.3\end{array}$ & $\begin{array}{l}36 \pm 3.0 \\
34 \pm 3.0\end{array}$ & $40 \pm 2.1$ & $21 \pm 3.2$ & $40 \pm 2.0$ & $25 \pm 1.0$ & $20 \pm 0.0$ & $25 \pm 1.6$ & $20 \pm 1.1$ & $26 \pm$ \\
\hline $\begin{array}{l}16 \\
1.3\end{array}$ & $\begin{array}{l}20 \pm 1.6 \\
20 \pm 1.6\end{array}$ & $\begin{array}{l}30 \pm 1.1 \\
30 \pm 2.0\end{array}$ & $40 \pm 2.3$ & $23 \pm 2.1$ & $60 \pm 3.0$ & $19 \pm 2.3$ & $20 \pm 1.1$ & $31 \pm 0.6$ & $20 \pm 0.0$ & $32 \pm$ \\
\hline $\begin{array}{l}18 \\
3.0\end{array}$ & $\begin{array}{l}20 \pm 1.0 \\
40 \pm 2.9\end{array}$ & $\begin{array}{l}29 \pm 3.6 \\
20 \pm 2.1\end{array}$ & $40 \pm 2.1$ & $21 \pm 3.1$ & $60 \pm 2.9$ & $18 \pm 2.6$ & $20 \pm 1.3$ & $20 \pm 0.0$ & $20 \pm 0.0$ & $31 \pm$ \\
\hline $\begin{array}{l}20 \\
2.9\end{array}$ & $\begin{array}{l}40 \pm 2.3 \\
40 \pm 0.0\end{array}$ & $\begin{array}{l}20 \pm 2.1 \\
19 \pm 1.1\end{array}$ & $60 \pm 3.0$ & $18 \pm 0.0$ & $80 \pm 2.0$ & $16 \pm 2.4$ & $20 \pm 0.0$ & $20 \pm 0.0$ & $40 \pm 2.1$ & $21 \pm$ \\
\hline
\end{tabular}

TABLE 3 (cont'd)

Var. excelsa

Concentration $(g / l)$ 
Harvest

\begin{tabular}{|c|c|c|c|c|c|c|c|c|c|c|}
\hline period & $\begin{array}{l}24 \\
72\end{array}$ & & 48 & & 72 & & 24 & & 48 & \\
\hline $\begin{array}{l}\text { (week) } \\
G T\end{array}$ & $\begin{array}{l}G \% \\
\quad G \%\end{array}$ & $\begin{array}{l}G T \\
\quad G T\end{array}$ & $G \%$ & $G T$ & $G \%$ & $G T$ & $G \%$ & $G T$ & $G \%$ & \\
\hline 10 & $\begin{array}{l}0 \pm 0.0 \\
0 \pm 0.0\end{array}$ & $\begin{array}{l}- \\
-\end{array}$ & $0 \pm 0.0$ & - & $0 \pm 0.0$ & - & $0 \pm 0.0$ & - & $0 \pm 0.0$ & - \\
\hline $\begin{array}{l}12 \\
0.0\end{array}$ & $\begin{array}{l}20 \pm 0.0 \\
20 \pm 1.3\end{array}$ & $\begin{array}{l}28 \pm 1.1 \\
22 \pm 0.7\end{array}$ & $20 \pm 1.1$ & $24 \pm 0.0$ & $0 \pm 0.0$ & $27 \pm 1.2$ & $0 \pm 0.0$ & - & $20 \pm 0.6$ & $20 \pm$ \\
\hline 14 & $20 \pm 0.6$ & $\begin{array}{l}27 \pm 1.3 \\
26+23\end{array}$ & $40 \pm 2.6$ & $25 \pm 1.1$ & $40 \pm 2.6$ & $36 \pm 1.3$ & $20 \pm 1.3$ & $30 \pm 1.9$ & $20 \pm 0.6$ & $27 \pm$ \\
\hline $\begin{array}{l}2.1 \\
16\end{array}$ & $\begin{array}{l}20 \pm 1.4 \\
40 \pm 3.0\end{array}$ & $26 \pm 1.3$ & $40 \pm 2.1$ & $17 \pm 1.7$ & $60 \pm 3.1$ & $29 \pm 2.1$ & $20 \pm 0.0$ & $26 \pm 1.1$ & $20 \pm 0.0$ & $31 \pm$ \\
\hline 1.3 & $40 \pm 2.1$ & $20 \pm 0.0$ & & & & & & & & \\
\hline $\begin{array}{l}18 \\
1.1\end{array}$ & $\begin{array}{l}60 \pm 3.3 \\
60 \pm 1.9\end{array}$ & $\begin{array}{l}17 \pm 1.0 \\
24 \pm 0.0\end{array}$ & $80 \pm 2.6$ & $18 \pm 1.1$ & $80 \pm 2.1$ & $15 \pm 0.7$ & $40 \pm 1.3$ & $22 \pm 1.3$ & $40 \pm 2.1$ & $26 \pm$ \\
\hline $\begin{array}{l}20 \\
2.0\end{array}$ & $\begin{array}{l}80 \pm 2.6 \\
80 \pm 1.4\end{array}$ & $\begin{array}{l}16 \pm 0.3 \\
18 \pm 1.1\end{array}$ & $80 \pm 0.6$ & $15 \pm 0.0$ & $80 \pm 3.6$ & $16 \pm 1.1$ & $40 \pm 1.3$ & $20 \pm 0.0$ & $80 \pm 1.7$ & $19 \pm$ \\
\hline
\end{tabular}

$-=$ There was no germination 60 days after sowing.

Key: $\mathrm{G} \%=$ Germination percentage; GT = Germination time (days).

TABLE 4

Effect of potassium nitrate $\left(\mathrm{KNO}_{3}\right)$ on percentage germination of seeds of fruits of Irvingia varieties plucked from forest floor (Mean + S.E)

Var. gabonensis

Concentration $(g / l)$
1.5

Hours of soaking

\section{Harvest}

period 24

(week) $\quad G \% \quad G T$

GT $\quad G \% \quad G T$

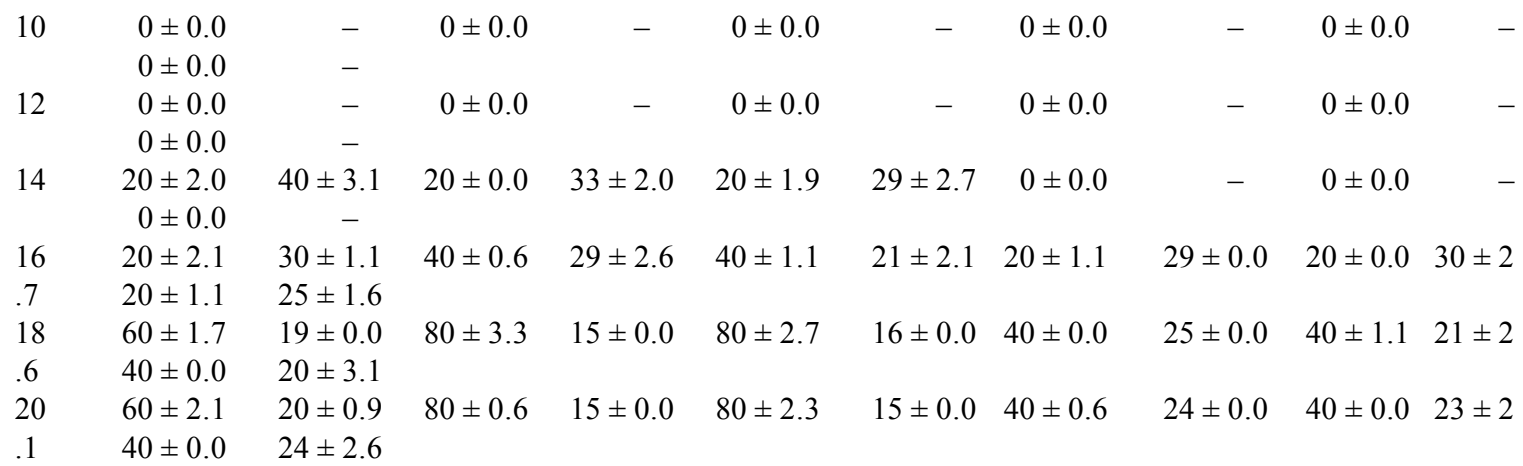

TABLE 4 (cont'd)

Var. excelsa 
Concentration $(g / l)$

$\begin{array}{ll}1.5 & 3.0\end{array}$

\begin{tabular}{|c|c|c|c|c|c|c|c|c|c|c|}
\hline Harves & & & & & & Hours o & soaking & & & \\
\hline period & $\begin{array}{c}24 \\
72\end{array}$ & & 48 & & 72 & & 24 & & 48 & \\
\hline $\begin{array}{l}\text { (week) } \\
G T\end{array}$ & $\begin{array}{l}G \% \\
G \%\end{array}$ & $\begin{array}{l}G T \\
\quad G T\end{array}$ & $G \%$ & $G T$ & $G \%$ & $G T$ & $G \%$ & $G T$ & $G \%$ & \\
\hline 10 & $\begin{array}{l}0 \pm 0.0 \\
0 \pm 0.0\end{array}$ & $\begin{array}{l}- \\
-\end{array}$ & $0 \pm 0.0$ & - & $0 \pm 0.0$ & - & $0 \pm 0.0$ & - & $0 \pm 0.0$ & - \\
\hline 12 & $20 \pm 0.0$ & $30 \pm 3.1$ & $40 \pm 1.0$ & $20 \pm 2.0$ & $20 \pm 0.0$ & $31 \pm 3.1$ & $20 \pm 0.0$ & $33 \pm 3.0$ & $20 \pm 1.4$ & $34 \pm 3$ \\
\hline .6 & $20 \pm 0.6$ & $37 \pm 5.1$ & & & & & & & & \\
\hline 14 & $40 \pm 2.0$ & $21 \pm 1.1$ & $40 \pm 2.1$ & $30 \pm 3.1$ & $40 \pm 1.1$ & $23 \pm 2.1$ & $20 \pm 0.0$ & $30 \pm 0.7$ & $40 \pm 2.1$ & $27 \pm 0$ \\
\hline .6 & $20 \pm 0.0$ & $30 \pm 1.1$ & & & & & & & & \\
\hline 16 & $60 \pm 2.1$ & $20 \pm 2.1$ & $60 \pm 1.6$ & $19 \pm 1.7$ & $60 \pm 2.6$ & $18 \pm 2.6$ & $20 \pm 0.6$ & $26 \pm 0.6$ & $40 \pm 2.3$ & $23 \pm 1$ \\
\hline .7 & $40 \pm 0.0$ & $27 \pm 0.0$ & & & & & & & & \\
\hline 18 & $60 \pm 2.0$ & $18 \pm 0.0$ & $80 \pm 2.5$ & $16 \pm 0.0$ & $80 \pm 3.0$ & $15 \pm 0.1$ & $20 \pm 0.0$ & $28 \pm 2.4$ & $40 \pm 1.1$ & $19 \pm 0$ \\
\hline .0 & $40 \pm 1.0$ & $18 \pm 0.0$ & & & & & & & & \\
\hline 20 & $60 \pm 1.3$ & $17 \pm 0.0$ & $80 \pm 2.9$ & $15 \pm 0.7$ & $80 \pm 0.0$ & $15 \pm 0.1$ & $40 \pm 0.1$ & $21 \pm 2.6$ & $40 \pm 0.0$ & $23 \pm 1$ \\
\hline .7 & $40 \pm 0.6$ & $25 \pm 2.6$ & & & & & & & & \\
\hline
\end{tabular}

Key: $\mathrm{G} \%=$ Germination percentage; GT $=$ Germination time (days).

The concentration $(3.0 \mathrm{~g} / \mathrm{l})$ of $\mathrm{KNO}_{3}$ solution used also affected the germinability. Hence, seeds soaked in $1.5 \mathrm{~g} / 1$ significantly $(P<0.05)$ enhanced germination potentials in both varieties and in the two harvests (Tables 3 and 4 ).

Mannitol treatment resulted in lower percentage germination $(40 \%)$ in both varieties and harvests throughout the developmental stages (Tables 5 and 6). However, prolonged soaking of seeds in mannitol significantly $(P<0.05)$ reduced germination potentials of the seeds of $I$. gabonensis with increased germination time. Seeds soaked in $3.0 \mathrm{~g} / 1$ for $72 \mathrm{~h}$ failed to germinate 60 days after sowing (DAS) (Tables 5 and 6).

TABLE 5

Effect of D - manitol on percentage germination of seeds of fruits of Irvingia varieties plucked from standing trees $($ Mean \pm S.E)

Var. gabonensis

1.5

3.0

Harvest

$\begin{array}{ll}\text { period } & 24 \\ & 72\end{array}$

(week) $\quad G \% \quad G T$

GT $\quad G \% \quad G T$

48

G\%

$G T$
Concentration $(\mathrm{g} / \mathrm{l})$

Hours of soaking

72

24

48

$G \%$

$G T$

G\%

$G T$

G\% 


\begin{tabular}{|c|c|c|c|c|c|c|c|c|c|c|}
\hline \multirow[t]{2}{*}{10} & $0 \pm 0.0$ & - & $0 \pm 0.0$ & - & $0 \pm 0.0$ & - & $0 \pm 0.0$ & - & $0 \pm 0.0$ & - \\
\hline & $0 \pm 0.0$ & - & & & & & & & & \\
\hline \multirow[t]{2}{*}{12} & $0 \pm 0.0$ & - & $0 \pm 0.0$ & - & $0 \pm 0.0$ & - & $0 \pm 0.0$ & - & $0 \pm 0.0$ & - \\
\hline & $0 \pm 0.0$ & - & & & & & & & & \\
\hline \multirow[t]{2}{*}{14} & $20 \pm 0.6$ & $31 \pm 1.6$ & $0 \pm 0.0$ & - & $0 \pm 0.0$ & - & $0 \pm 0.0$ & - & $0 \pm 0.0$ & - \\
\hline & $0 \pm 0.0$ & - & & & & & & & & \\
\hline \multirow[t]{2}{*}{16} & $20 \pm 1.2$ & $24 \pm 1.7$ & $20 \pm 0.0$ & $29 \pm 1.2$ & $20 \pm 0.0$ & $20 \pm 1.1$ & $0 \pm 0.0$ & - & $0 \pm 0.0$ & - \\
\hline & $20 \pm 1.1$ & $36 \pm 1.7$ & & & & & & & & \\
\hline 18 & $40 \pm 1.8$ & $27 \pm 3.1$ & $20 \pm 0.0$ & $27 \pm 3.1$ & $40 \pm 1.0$ & $28 \pm 2.1$ & $20 \pm 0.0$ & $31 \pm 3.1$ & $20 \pm 0.7$ & $20 \pm$ \\
\hline 1.1 & $0 \pm 0.0$ & - & & & & & & & & \\
\hline 20 & $40 \pm 0.3$ & $26 \pm 2.7$ & $40 \pm 0.0$ & $22 \pm 0.0$ & $40 \pm 1.7$ & $30 \pm 2.6$ & $40 \pm 0.9$ & $26 \pm 2.1$ & $40 \pm 0.6$ & $26 \pm$ \\
\hline 2.6 & $0 \pm 0.0$ & - & & & & & & & & \\
\hline
\end{tabular}

TABLE 5 (cont'd)

Var. excelsa

Concentration $(g / l)$

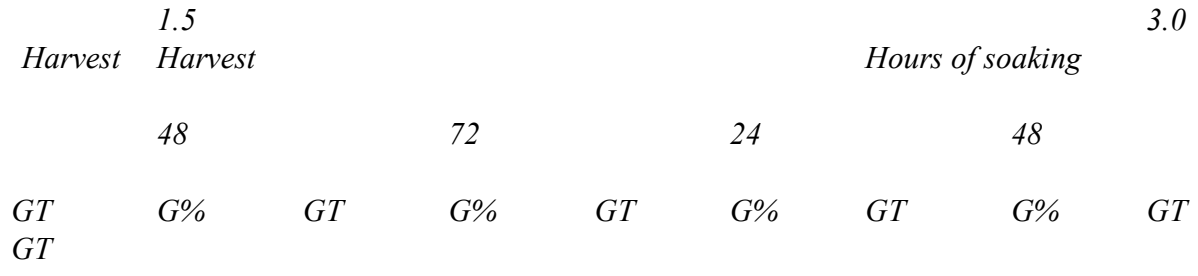

\begin{tabular}{|c|c|c|c|c|c|c|c|c|c|c|}
\hline 10 & $\begin{array}{c}0 \pm 0.0 \\
0 \pm 0.0\end{array}$ & ${ }_{-}^{-}$ & $0 \pm 0.0$ & - & $0 \pm 0.0$ & - & $0 \pm 0.0$ & - & $0 \pm 0.0$ & - \\
\hline 12 & $\begin{array}{c}0 \pm 0.0 \\
0 \pm 0.0\end{array}$ & - & $0 \pm 0.0$ & - & $0 \pm 0.0$ & - & $0 \pm 0.0$ & - & $0 \pm 0.0$ & - \\
\hline 14 & $20 \pm 0.0$ & $26 \pm 0.0$ & $20 \pm 0.0$ & $24 \pm 0.0$ & $20 \pm 0.7$ & $31 \pm 1.6$ & $20 \pm 2.1$ & $38 \pm 0.0$ & $20 \pm 1.6$ & $26 \pm$ \\
\hline 1.2 & $20 \pm 0.6$ & $31 \pm 3.6$ & & & & & & & & \\
\hline 16 & $20 \pm 0.0$ & $31 \pm 2.3$ & $40 \pm 1.1$ & $24 \pm 1.6$ & $20 \pm 0.1$ & $26 \pm 0.0$ & $40 \pm 2.0$ & $20 \pm 0.0$ & $40 \pm 2.1$ & $30 \pm$ \\
\hline 2.1 & $20 \pm 2.3$ & $28 \pm 1.1$ & & & & & & & & \\
\hline 18 & $20 \pm 0.0$ & $23 \pm 3.6$ & $40 \pm 1.1$ & $21 \pm 2.7$ & $20 \pm 0.1$ & $34 \pm 3.6$ & $40 \pm 0.6$ & $20 \pm 0.0$ & $40 \pm 2.3$ & $22 \pm$ \\
\hline 1.1 & $0 \pm 0.0$ & - & & & & & & & & \\
\hline 20 & $40 \pm 0.0$ & $23 \pm 2.1$ & $40 \pm 2.0$ & $24 \pm 2.7$ & $0 \pm 0.0$ & $0 \pm 0.0$ & $40 \pm 0.4$ & $23 \pm 2.1$ & $40 \pm 0.0$ & $25 \pm$ \\
\hline 0.0 & $0 \pm 0.0$ & - & & & & & & & & \\
\hline
\end{tabular}

$-=$ There was no germination 60 days after sowing.

Key: $\mathrm{G} \%=$ Germination percentage; GT $=$ Germination time (days).

TABLE 6

Effect of D - manitol on percentage germination of seeds of fruits of Irvingia varieties plucked from forest floor (Mean \pm S.E)

Var. gabonensis

Concentration $(g / l)$

15

30

Harvest

period

24

48

72

Hours of soaking

(week)

$\begin{array}{lllll}24 & & 48 & & 72 \\ & 72 & & & \\ G T & G T & G \% & G T & G \% \\ G T & G \% & & & \end{array}$

24

48

$G T \quad G \%$

$G T \quad G \%$ 


\begin{tabular}{|c|c|c|c|c|c|c|c|c|c|}
\hline 10 & $0 \pm 0.0$ & - & $0 \pm 0.0$ & - & $0 \pm 0.0$ & - & $0 \pm 0.0$ & - & $0 \pm 0.0$ \\
\hline- & $0 \pm 0.0$ & & & & & & & & \\
\hline 12 & $20 \pm 1.7$ & $31 \pm 1.1$ & $20 \pm 1.3$ & $27 \pm 2.6$ & $0 \pm 0.0$ & - & $0 \pm 0.0$ & - & $0 \pm 0.0$ \\
\hline- & $0 \pm 0.0$ & & & & & & & & \\
\hline 14 & $20 \pm 0.0$ & $34 \pm 3.0$ & $40 \pm 1.7$ & $27 \pm 0.0$ & $20 \pm 2.3$ & $36 \pm 4.1$ & $0 \pm 0.0$ & - & $0 \pm 0.0$ \\
\hline- & $0 \pm 0.0$ & & & & & & & & \\
\hline 16 & $40 \pm 2.1$ & $22 \pm 2.1$ & $20 \pm 0.3$ & $26 \pm 2.0$ & $20 \pm 2.1$ & $20 \pm 1.0$ & $20 \pm 0.1$ & $20 \pm 0.7$ & $20 \pm 0.7$ \\
\hline $32 \pm 2.4$ & $20 \pm 0.0$ & & & & & & & & \\
\hline 18 & $40 \pm 2.1$ & $16 \pm 0.0$ & $40 \pm 3.0$ & $22 \pm 3.0$ & $20 \pm 1.1$ & $26 \pm 0.0$ & $40 \pm 0.0$ & $29 \pm 3.6$ & $20 \pm 0.0$ \\
\hline $31 \pm 2.1$ & $20 \pm 1.1$ & & & & & & & & \\
\hline 20 & $40 \pm 1.1$ & $17 \pm 1.1$ & $40 \pm 1.3$ & $20 \pm 1.2$ & $20 \pm 2.0$ & $34 \pm 1.3$ & $40 \pm 0.0$ & $27 \pm 2.1$ & $40 \pm 0.0$ \\
\hline $29 \pm 1.1$ & $20 \pm 0.7$ & & & & & & & & \\
\hline
\end{tabular}

TABLE 6 (cont'd)

Var. excelsa

Concentration $(g / l)$

1.5

3.0

Harvest

$-48$

48

Hours of soaking

$\begin{array}{lll} & 72 & \\ \text { (week) } & G \% & G T \\ G T & G \% & G T\end{array}$

$G \% \quad G T$

24

48

10

$$
\begin{aligned}
& 0 \pm 0.0 \\
& 0 \pm 0.0
\end{aligned}
$$

$12 \quad 0 \pm 0.0$

$0 \pm 0.0$

$14 \quad 20 \pm 0.1$

$20 \pm 0.0$

$16 \quad 20 \pm 1.1$

$.6 \quad 20 \pm 0.0$

$18 \quad 40 \pm 0.0$

$.0 \quad 0 \pm 0.0$

$20 \quad 40 \pm 0.0$

\begin{tabular}{|c|c|c|c|c|c|c|c|c|}
\hline- & $0 \pm 0.0$ & - & $0 \pm 0.0$ & - & $0 \pm 0.0$ & - & $0 \pm 0.0$ & - \\
\hline $\begin{array}{l}- \\
- \\
-\end{array}$ & $20 \pm 2.0$ & $31 \pm 3.5$ & $20 \pm 0.0$ & $29 \pm 1.1$ & $0 \pm 0.0$ & - & $0 \pm 0.0$ & \\
\hline $\begin{array}{l}26 \pm 1.7 \\
25 \pm 2.3\end{array}$ & $20 \pm 1.3$ & $24 \pm 0.7$ & $20 \pm 0.9$ & $24 \pm 1.2$ & $0 \pm 0.0$ & - & $0 \pm 0.0$ & - \\
\hline $\begin{array}{l}22 \pm 0.3 \\
20 \pm 2.1\end{array}$ & $20 \pm 0.4$ & $24 \pm 2.1$ & $40 \pm 2.3$ & $19 \pm 2.1$ & $20 \pm 0.0$ & $22 \pm 2.1$ & $20 \pm 0.0$ & $29 \pm 3$ \\
\hline $\begin{array}{c}26 \pm 3.8 \\
-\end{array}$ & $40 \pm 1.1$ & $30 \pm 2.7$ & $20 \pm 2.1$ & $36 \pm 3.7$ & $40 \pm 0.0$ & $21 \pm 2.7$ & $40 \pm 2.0$ & $21 \pm 0$ \\
\hline $24 \pm 0.7$ & $40 \pm 2.1$ & $31 \pm 3.1$ & $20 \pm 2.0$ & $30 \pm 2.7$ & $40 \pm 0.0$ & $30 \pm 3.6$ & $40 \pm 2.0$ & 231 \\
\hline
\end{tabular}

$.1 \quad 0 \pm 0.0$

- = There was no germination 60 days after sowing.

Key: $\mathrm{G} \%=$ Germination percentage; $\mathrm{GT}=$ Germination time (days).

Generally, there was an increase in germinability $(60 \%)$ of seeds soaked in polyethylene glycol (PEG) as compared to those in mannitol in both varieties and harvests between 18th and 20th WAF (Tables 7 and 8). Seeds soaked in concentration of $10.0 \mathrm{~g} / \mathrm{l}$ recorded $60 \%$ germination in both varieties and harvests between the 16 and 20 WAF (Tables 7 and 8). PEG treatment also significantly $(P<0.05)$ reduced germination time of both varieties in both harvests (Tables 7 and 8).

TABLE 7

Effect of polyethylene glycol (PEG) on percentage germination of seeds of fruits of Irvingia varieties plucked from standing trees (Mean + S.E)

Var. gabonensis 


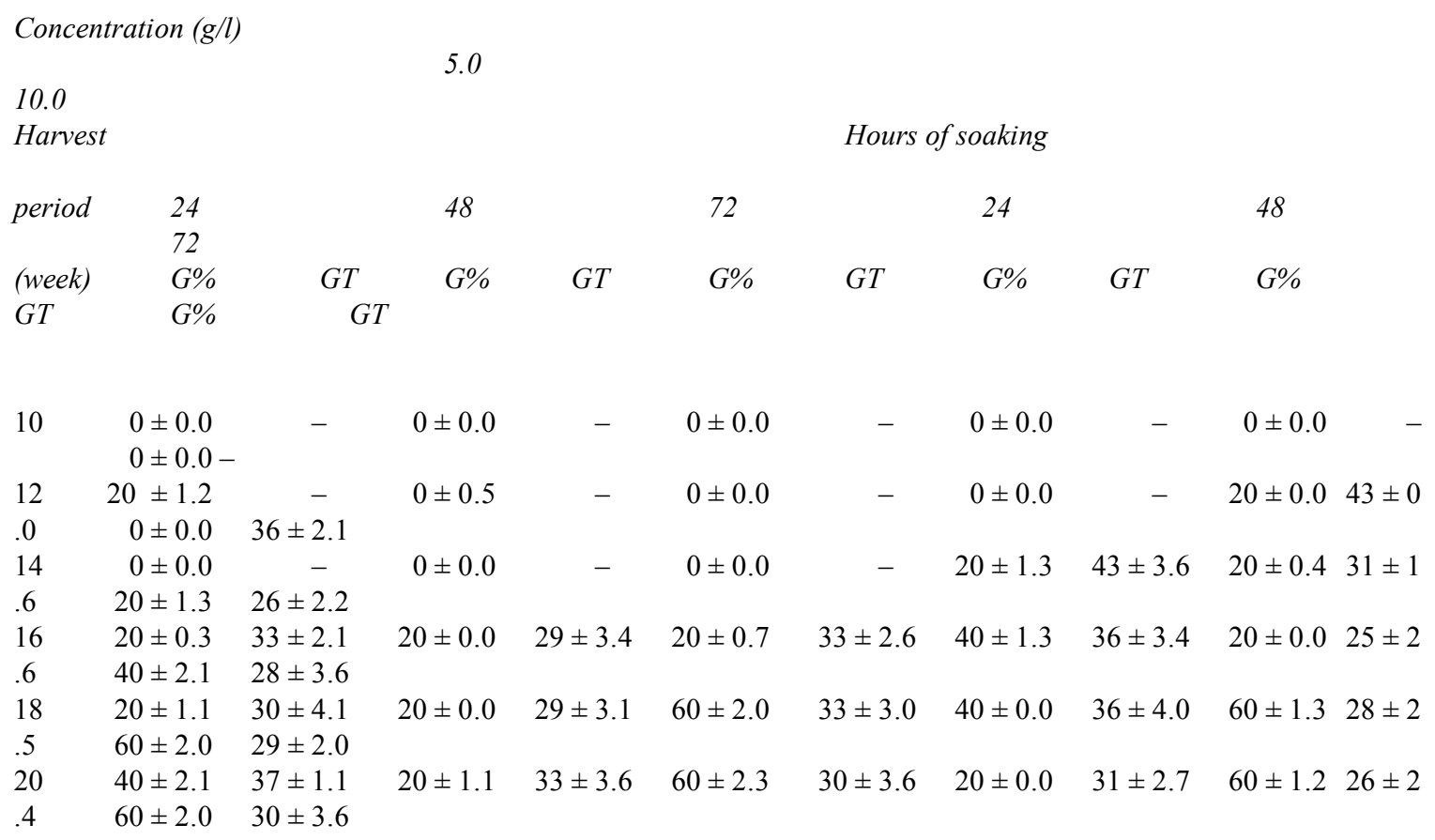

TABLE 7 (cont'd)

Var. excelsa

Concentration $(g / l)$

1.5

Harvest

3.0

\begin{tabular}{|c|c|c|c|c|c|c|c|c|c|c|}
\hline period & $\begin{array}{l}24 \\
72\end{array}$ & & 48 & & 72 & & 24 & & 48 & \\
\hline (week) & $\begin{array}{l}G \% \\
G \%\end{array}$ & $\begin{array}{l}G T \\
G T\end{array}$ & $G \%$ & $G T$ & $G \%$ & $G T$ & $G \%$ & $G T$ & $G \%$ & $G T$ \\
\hline 10 & $\begin{array}{l}0 \pm 0.0 \\
0 \pm 0.0\end{array}$ & $\begin{array}{l}- \\
-\end{array}$ & $0 \pm 0.0$ & - & $0 \pm 0.0$ & - & $0 \pm 0.0$ & - & $0 \pm 0.0$ & - \\
\hline 12 & $\begin{array}{l}0 \pm 0.0 \\
0 \pm 0.0\end{array}$ & $\begin{array}{l}- \\
-\end{array}$ & $20 \pm 2.0$ & $31 \pm 3.5$ & $20 \pm 0.0$ & $29 \pm 1.1$ & $0 \pm 0.0$ & - & $0 \pm 0.0$ & - \\
\hline 14 & $\begin{array}{l}20 \pm 0.1 \\
20 \pm 0.0\end{array}$ & $\begin{array}{l}26 \pm 1.7 \\
25 \pm 2.3\end{array}$ & $20 \pm 1.3$ & $24 \pm 0.7$ & $20 \pm 0.9$ & $24 \pm 1.2$ & $0 \pm 0.0$ & - & $0 \pm 0.0$ & - \\
\hline $\begin{array}{l}16 \\
.6\end{array}$ & $\begin{array}{l}20 \pm 1.1 \\
20 \pm 0.0\end{array}$ & $\begin{array}{l}22 \pm 0.3 \\
20 \pm 2.1\end{array}$ & $20 \pm 0.4$ & $24 \pm 2.1$ & $40 \pm 2.3$ & $19 \pm 2.1$ & $20 \pm 0.0$ & $22 \pm 2.1$ & $20 \pm 0.0$ & $29 \pm 3$ \\
\hline $\begin{array}{l}18 \\
.0\end{array}$ & $\begin{aligned} 40 & \pm 0.0 \\
0 & \pm 0.0\end{aligned}$ & $\begin{array}{c}26 \pm 3.8 \\
-\end{array}$ & $40 \pm 1.1$ & $30 \pm 2.7$ & $20 \pm 2.1$ & $36 \pm 3.7$ & $40 \pm 0.0$ & $21 \pm 2.7$ & $40 \pm 2.0$ & $21 \pm 0$ \\
\hline 20 & $\begin{array}{r}40 \pm 0.0 \\
0 \pm 0\end{array}$ & $24 \pm 0.7$ & $40 \pm 2.1$ & $31 \pm 3.1$ & $20 \pm 2.0$ & $30 \pm 2.7$ & $40 \pm 0.0$ & $30 \pm 3.6$ & $40 \pm 2.0$ & 231 \\
\hline
\end{tabular}

$-=$ There was no germination 60 days after sowing.

Key: $\mathrm{G} \%=$ Germination percentage; $\mathrm{GT}=$ Germination time (days). 
TABLE 8

Effect of polyethylene glycol (PEG) on percentage seed germination of fruits of Irvingia varieties plucked from forest floor (Mean + S.E)

Var. gabonensis

Concentration $(g / l)$

5.0

10.0

Harvest

period 24

(week) $\quad G \% \quad G T$

$G \% \quad G T$
48

G\%

$0 \pm 0.0$

$20 \pm 0$

$40 \pm 0.0 \quad 24 \pm 2.0$

$40 \pm 2.0 \quad 28 \pm 2.0$

$20 \pm 2.3 \quad 29 \pm 3.6$

$60 \pm 1.6 \quad 21 \pm 0.0$
Hours of soaking

\begin{tabular}{|c|c|c|c|c|}
\hline 72 & & 24 & & 48 \\
\hline$G \%$ & $G T$ & $G \%$ & $G T$ & $G \%$ \\
\hline
\end{tabular}

$\begin{array}{cccccc}20 \pm 0.0 & 34 \pm 1.6 & 0 \pm 0.0 & - & 0 \pm 0.0 & - \\ 20 \pm 1.1 & 36 \pm 2.1 & 20 \pm 0.6 & 31 \pm 2.3 & 20 \pm 0.7 & 40 \pm \\ 40 \pm 1.3 & 27 \pm 1.1 & 20 \pm 0.0 & 31 \pm 3.1 & 40 \pm 1.2 & 29 \pm \\ 40 \pm 1.4 & 25 \pm 1.9 & 40 \pm 0.1 & 28 \pm 2.0 & 60 \pm 0.6 & 21 \pm \\ 60 \pm 1.7 & 18 \pm 1.0 & 40 \pm 0.0 & 29 \pm 2.3 & 60 \pm 2.1 & 18 \pm \\ 60 \pm 0.1 & 20 \pm 0.2 & 60 \pm 2.1 & 21 \pm 3.1 & 60 \pm 1.1 & 18 \pm\end{array}$

TABle 8

Var. excelsa

Concentration $(g / l)$

5.0

Hours of soaking

Harvest

$\begin{array}{clllllllll}\text { period } & 24 & & 48 & & 72 & & 24 & & 48 \\ \text { (week) } & G \% & G T & G \% & G T & G \% & G T & G \% & G T & G \% \\ & G T & G \% & G T & & & & & \end{array}$

$\begin{array}{lrccccccccc}10 & 0 \pm 0.0 & - & 0 \pm 0.0 & - & 0 \pm 0.0 & - & 0 \pm 0.0 & - & 0 \pm 0.0 & \\ - & 0 \pm 0.0 & - & & & & & & & & \\ 12 & 20 \pm 0.0 & 29 \pm 2.3 & 20 \pm 0.1 & 21 \pm 2.1 & 20 \pm 0.0 & 43 \pm 4.6 & 20 \pm 0.0 & 21 \pm 3.1 & 20 \pm 0.0 & 23 \\ \pm 2.0 & 20 \pm 1.1 & 31 \pm 2.1 & & & & & & & & \\ 14 & 20 \pm 0.0 & 33 \pm 0.7 & 20 \pm 0.0 & 36 \pm 3.6 & 40 \pm 1.1 & 23 \pm 2.8 & 20 \pm 0.0 & 29 \pm 3.6 & 20 \pm 0.0 & 28 \\ \pm 2.8 & 40 \pm 0.0 & 24 \pm 2.0 & & & & & & & & \\ 16 & 20 \pm 0.7 & 28 \pm 0.6 & 40 \pm 1.2 & 31 \pm 3.9 & 60 \pm 0.0 & 37 \pm 2.6 & 40 \pm 1.2 & 24 \pm 2.5 & 40 \pm 0.0 & 26 \\ \pm 2.6 & 60 \pm 0.0 & 46 \pm 0.7 & & & & & & & & \\ 18 & 20 \pm 0.0 & 28 \pm 2.1 & 40 \pm 0.0 & 31 \pm 0.9 & 60 \pm 0.0 & 34 \pm 3.1 & 20 \pm 0.0 & 23 \pm 2.6 & 40 \pm 0.0 & 26 \\ \pm 2.2 & 60 \pm 0.0 & 16 \pm 1.6 & & & & & & & & \\ 20 & 20 \pm 0.6 & 21 \pm 2.0 & 40 \pm 0.0 & 28 \pm 2.1 & 60 \pm 0.0 & 25 \pm 3.0 & 40 \pm 1.0 & 20 \pm 0.7 & 60 \pm 0.0 & 16 \\ \pm 0.0 & 60 \pm 0.0 & 14 \pm 0.0 & & & & & & & & \end{array}$

- $=$ There was no germination 60 days after sowing. 
Key: $\mathrm{G} \%=$ Germination percentage; $\mathrm{GT}=$ Germination time (days).

\section{Discussion}

The study has shown that Irvingia gabonensis trees produce both physiolo-gically immature and mature fruits with varying degrees of viability which increases with age throughout the developmental stages. This finding shows that seeds of fruits that are harvested during the first 10 weeks of fruiting are immature, not viable and lack capacity to germinate because they are still undergoing stages of embryo develop-ment. The fruits which assume ripening characteristics (yellowing) and are palatable shortly after harvest on standing trees and from the forest floor may be obtained from the 14th to 20th week after fruiting.

The low germination capacity recorded in I. gabonensis could be due to the recalcitrant nature of the seeds which, unlike orthodox seeds, do not conform with the rule of increasing longevity with fall in temperature and moisture content (Roberts, 1973; Bishnoi, 1974; Ellis et al., 1989; Ellis et al., 1990a; Omokaro et al., 1999). Such seeds are produced by large-seeded plant species. Also, most tropical fruit crops like cocoa, mango and citrus have been identified to produce recalcitrant seeds (Chin, 1980; Aiyelaagbe, 1994; Awodoyan \& Ogbonnaya, 1994). Nya et al. (2000) also reported that I. gabonensis seeds exhibit poor germination potential with less than 50\% germination capacity. Oni (1984), working on some tropical forest trees, reported similar germinability (40\%). This study has shown that fruits of Irvingia harvested from standing trees and forest floor 16th-20th week after fruiting, respectively, contain seeds that have attained a measure of physiological maturity and germination capacity of $20 \%$ which can be enhanced to $80 \%$ following pretreatments. However, seeds harvested 18th-20th week after fruiting contain mature seeds capable of $80 \%$ germinability which was confirmed by TZ staining (Moore, 1973). This finding shows that viable seeds can be obtained from standing trees of I. gabonensis and also picked from forest floor for seedlings production.

The increase in germination capacity (80\%) of seeds soaked in $1.5 \mathrm{~g} / 1$ of $\mathrm{KNO}_{3}$ for 48 and 72 $\mathrm{h}$ could be due to increase in embryo length as Asiegbu \& Onugua (1994) had noted that 1-3 g/l $\mathrm{KNO}_{3}$ increased the embryo length of Nsukka yellow pepper. The depression in germination of seeds with $3.0 \mathrm{~g} / 1$ of $\mathrm{KNO}_{3}$ was probably due to toxicity of the salt solution to the seeds. Asiegbu \& Onugua (1994) also noted that $5 \mathrm{~g} / 1$ of $\mathrm{KNO}_{3}$ was toxic to African spinach seeds.

PEG significantly $(P<0.05)$ increased percentage germination of Irvingia seeds in both harvests. Increase in germination $(60 \%)$ with seeds soaked for $72 \mathrm{~h}$ could be due to increase in embryo length. A similar result was obtained by Black (1972) who reported that PEG increased the embryo and hypocotyl length in green-grams seeds. Decrease in germination of seeds soaked for $24 \mathrm{~h}$ was probably due to less penetration of PEG solution into the internal part of the seed. A similar result was obtained by Black (1972) who reported that hard testa of many seeds acts as hindrance to water penetration. The significant increase in the germination capacity with $\mathrm{KNO}_{3}$ and PEG confirms the report of Thompson \& Horn (1944) who reported that some chemicals and growth regulators can improve the germination of lettuce seeds.

Mannitol decreased the percentage germination of Irvingia seeds with increased concentrations. The none germination recorded in seeds soaked for $72 \mathrm{~h}$ in $3.0 \mathrm{~g} / \mathrm{l}$ mannitol could be due to inducing drought condition to the seeds. Similar reports were made by Roberts \& King (1980) and Deijode (1983) who noted that treatment of recalcitrant seeds with high percentage mannitol resulted in the loss of viability. Khan (1980) and Esenowo (1995) also noted similar reduction in germination and growth with mannitol.

The present study has shown that seed maturity is positively related to moisture content, viability and germination, and viability can be determined by means of TZ staining (Moore, 1973). 
Since seeds of fruits harvested from standing trees and forest floor attain viability between the 14th and 20th week after fruiting and maximum germination potential of the seeds could be enhanced with the use of pretreatments, it is recommended that nursery operators should explore these methods as a source of seedling regeneration after determination of physiological maturity by using TZ staining.

\section{References}

Adeyoju S. K. and Enabor E. E. (1985). Nigerian Forest Reserve System II. A review of the tariff structure and proposals for changes on timber poles, fuel wood and minor products. FORMECU Report, Federal Department of Forestry, Ibadan, Nigeria.

Aiyelaagbe I. O. O. (1988). Effective nursery management as a tool for boosting the production of fruit trees. Paper presented at the 10th Annual Conference of Horticultural Society of Nigeria held at Okada Wonderland, Nigeria, 6th-11th November 1988.

Asiegbu J. and Onugua A. (1994). Effects of seed priming with different chemicals on the germination of African spinach and Nsukka yellow pepper seeds. Nig. J. Bot. 7: 63-67.

Awodoyin R. and Ogbonnaya F. (1994). Studies on some nursery management techniques of Irvingia gabonensis var. excelsa. Paper presented at the ICRAF workshop on Irvingia gabonensis, 9-11 May, 1994 IITA Ibadan Nigeria.

Bishnoi W. R. (1974). Physiological maturity of seeds in Triticade hexaploid L. Crop Sci. 14: 819-821.

Black M. (1972). Control Process in Germination and Dormancy. (J. J. Head and E. O. Lowenster, ed.), pp. 1-9. Oxford Biology Reader No. 20.

Chin H. F. (1980). Germination in recalcitrant crop seeds. (H. F. Chin and E. H. Roberts, ed.). Kuala Lumpur Tropical Press SDN, BHD.

Deijode S. D. (1983). Studies on vigour and viability of seeds at different stages of fruit development in tomato. Singapore J. Prim. Ind. 2: 106-109.

Donna M. A., Ogar A. E. and Otu M. (1994). A user survey on Irvingia gabonensis in Cross River State, Nigeria: Local Storage Methods of Bush Mango. Paper presented at the pre-germplasm collection meeting on Irvingia gabonensis organized by ICRAF, Nairobi, Kenya held at IIITA, Ibadan, Nigeria, May 1994.

Ellis R. H., Hong T. D. and Roberts E. H. (1987). The development of desiccation-tolerance and maximum seed quality during seed maturation in six grain legumes. Ann. Bot. 59: 23-29.

Ellis R. H., Hong T. D., Roberts E. H. and Tao K. L. (1990a). Low moisture content limits relations between seed longevity and moisture. Ann. Bot. 64: 493-504.

Esenowo G. I. (1995). Germination ecology of lactuca sativa var. madrilène. West Af. J. Biol. appl. Chem. 40: 33-39.

International Seed Testing Association (1985a). International rules for testing Rules 1985. Seed Sci. Technol. 13: 299-355.

International Seed Testing Association (1985b). International rules for testing Annexes 1985. Seed Sci. Technol. 13: $356-513$.

Khan A. A. (1980). The Physiological and Biochemistry of seed Dormancy and Germination. North Holland Publishing Company. Amsterdam, New York. 447 pp.

Moore R. P. (1973). Tetrazolium staining for assessing seed quality. In Seed Ecology. (W. Heydecker, ed.), pp. 347366. London, Butterworths.

Nya P. J. (1997). Comparative studies on seed morphology, seed germination and early seedling development in two varieties of bush mango (Irvingia gabonensis var. excelsa and var. gabonensis) (MSc. Thesis.). University of Calabar, Calabar, Nigeria.

Nya P. J., Omokaro D. N. and Nkang A. E. (2000). Comparative studies of seed morphology, moisture content and seed germination of two varieties of Irvingia gabonensis. Global J. pure appl. Sci. 6(3): 375-378.

Okafor J. C. (1989). Agro forestry aspect. A study prepared by worldwide fund for nature for Rross River National Park Project (ODA assisted). Forestry Department, Headquarters, Calabar, Nigeria.

Okafor J. C. (1990). Development and Selection of Commercial Viable Cultivars from Forest Species of fruit. Proceedings of the Twelveth Plenary Meeting of Act Fat. Symposium, I. Hamburg, 1990.

Okafor J. C., Omorondion F. I. and Amaza F. (1994). Non-Timber Forest Products: Draft Report prepared for the National Tropical Forestry Action Programme (NTFAP). Forestry Management, Evaluation and Co-operation Unit (FORMECU), Federal Department of Forestry, Abuja, Nigeria.

Omokaro D. N., Nkang A. and Nya P. J. (1999). Effects of desiccation and subsequent rehydration on the germination of Irvingia gabonensis var. excelsa seeds. Seed Sci. Technol. 27: 877-884. 
Omoluabi A. C. (1994). Trade in Timber and Non-Timber Forest Products in Cross River State of Nigeria. Report prepared for the Cross River State Forestry Project (ODA assisted). Forestry Department, Headquarters, Calabar, Nigeria.

Oni O. (1984). Conservating the genetic resources of indigenous West African hardwoods by vegetative propagation in Nigeria. 12th Annual Conference of the Genetics Society of Nigeria, Ibadan. 3rd-7th December, 1984.

Roberts E. H. and King M. W. (1980). The Characteristics of Recalcitrant seeds. In Recalcitrant crop seeds. (H. F. Chin and E. H. Roberts, ed.) Kuala Lumpur. Tropical Pres SND. BHD.

Thompson R. C. and Horn N. C. (1944). Germination of lettuce at a high temperature $\left(25-35^{\circ} \mathrm{C}\right)$ stimulated by

thiourea. Proc. Am. Chem. Soc. 77: 1932. 